\title{
Generalised Control-Oriented Modelling Framework for Multi-Energy Systems
}

DOI:

10.1016/j.apenergy.2018.10.074

\section{Document Version}

Accepted author manuscript

Link to publication record in Manchester Research Explorer

\section{Citation for published version (APA):}

Long, S., Marjanovic, O., \& Parisio, A. (2019). Generalised Control-Oriented Modelling Framework for Multi-Energy Systems. Applied Energy, 235, 320-331. https://doi.org/10.1016/j.apenergy.2018.10.074

\section{Published in:}

Applied Energy

\section{Citing this paper}

Please note that where the full-text provided on Manchester Research Explorer is the Author Accepted Manuscript or Proof version this may differ from the final Published version. If citing, it is advised that you check and use the publisher's definitive version.

\section{General rights}

Copyright and moral rights for the publications made accessible in the Research Explorer are retained by the authors and/or other copyright owners and it is a condition of accessing publications that users recognise and abide by the legal requirements associated with these rights.

\section{Takedown policy}

If you believe that this document breaches copyright please refer to the University of Manchester's Takedown Procedures [http://man.ac.uk/04Y6Bo] or contact uml.scholarlycommunications@manchester.ac.uk providing relevant details, so we can investigate your claim.

\section{OPEN ACCESS}




\title{
Generalised Control-Oriented Modelling Framework for Multi-Energy Systems
}

\author{
Sebastian Long, Ognjen Marjanovic and Alessandra Parisio \\ School of Electrical and Electronic Engineering \\ University of Manchester
}

\begin{abstract}
A requirement for new sources of flexibility in meeting energy demand and the growing interest in the interaction between energy sectors have drawn attention to multienergy systems, which can offer numerous benefits, e.g. providing flexibility to counteract the intermittency of renewable generation and increase energy efficiency. In order to maximise these benefits, advanced control methods are required. The model predictive control methodology is a promising candidate for such applications as it is capable of incorporating economic and operational objectives whilst respecting various technical, regulatory and environmental constraints. In order to implement such a control strategy effectively, it is necessary to develop appropriate system models. This paper presents a novel generalised modelling framework for multi-energy systems that is particularly well suited, though not limited to, predictive control applications. The proposed approach is capable of representing energy converter arrangements of arbitrary complexity containing multiple energy vectors, as well as multi-directional energy flow, multi-generation and multi-mode devices, a wide range of controllable producers/consumers, energy storage and flexible loads. The effectiveness of the approach is demonstrated using a representative case study based on three buildings at the University of Manchester where the developed model is incorporated into a model predictive control scheme. The simulation results show how the controller minimises the cost of purchasing energy whilst satisfying operational constraints, and managing various types of flexible demand.
\end{abstract}

Index Terms-Model Predictive Control, control-oriented mod-

elling, multi-energy systems, demand-side management

\section{NOMENCLATURE}

$k \quad$ Sampling period

$h \quad$ Scheduling period

$n_{i}, n_{j} \quad$ Node indices

$\eta_{\left(n_{i} n_{j}\right)} \quad$ Conversion factor from node $n_{i}$ to $n_{j}$

$P_{\left(n_{i} \rightarrow n_{j}\right)} \quad$ Power flow from node $n_{i}$ to node $n_{j}$

$P_{n_{i}} \quad$ Power flow in/out of node $n_{i}$

$\mathcal{I}_{\rightarrow n_{l}} \quad$ Index set of source nodes that connect to $n_{l}$

$\mathcal{I}_{n_{l} \rightarrow} \quad$ Index set of sink nodes that $n_{l}$ connects to

$\delta_{\left(n_{i} \rightarrow n_{j}\right)} \quad$ Binary decision variable associated with arc $n_{i} n_{j}$

$\mathrm{x} \quad$ State vector

$\mathbf{u} \quad$ Input vector

A State transition matrix

B Input matrix

C Output matrix

D Feed-forward matrix

Authors acknowledge financial support from EPSRC (EP/L016141/1) through the EPSRC Centre for Doctoral Training in Power Networks $v$

$\mathbf{w}$

$y$

$p_{j, n_{i}}$

$P_{p_{j, n_{i}}}$

$G_{m}$

$L_{n}$

$N_{G, p_{j, n_{i}}}$

$N_{L, p_{j, n_{i}}}$

$N_{H}$

$N_{n}^{i}$

$l_{n, i}$

$\tilde{l}_{n, i}$

$\Delta l_{n, i}^{+}$

$\Delta l_{n, i}^{-} \quad$ Slack variable relating to the amount of cur-

Storage model charging/discharging input

Storage model input disturbance vector

Storage model output state

Prosumer $j$ connected to node $n_{i}$

Net power demand/consumption of prosumer

$p_{j, n_{i}}$

$m^{t h}$ prosumer generating component

$n^{\text {th }}$ prosumer load component

Number of generating components connected to prosumer $p_{j, n_{i}}$

Number of load components connected to prosumer $p_{j, n_{i}}$

Scheduling/control horizon

Number of energy segments for demand $n$

Actual energy consumption of $i^{\text {th }}$ segment of demand $n$

Energy consumption based on the nominal energy requirement for the $i^{t h}$ segment of demand $n$

Slack variable relating to the amount of scheduled energy in excess of the nominal demand for the $i^{t h}$ segment of demand $n$ tailed energy from the nominal demand for the $i^{\text {th }}$ segment of demand $n$

$E_{n, i} \quad$ Total nominal energy requirement of the $i^{t h}$ segment of demand $n$

$\delta_{n, i}^{p}$

$\delta_{n, i}^{c}$

$\delta_{n, i}^{w}$

$L_{n}$

Binary variable to indicate whether or not ( 1 or 0 ) the $i^{\text {th }}$ segment of demand $n$ is processing Binary variable to indicate whether or not (1 or 0 ) the $i^{\text {th }}$ segment of demand $n$ is complete Binary variable to indicate whether or not (1 or 0 ) the $i^{\text {th }}$ segment of demand $n$ is waiting Resulting energy consumption of demand $n$ accounting for contributions from all energy segments

$\mathbf{T}_{n}^{p} \quad$ Energy user's time-preference vector

$S_{n_{i}} \quad$ Standby efficiency of storage device connected to node $n_{i}$

$Q_{n_{i}} \quad$ Charging/discharging power of storage device connected to node $n_{i}$

$R_{n_{i}} \quad$ Energy stored in device

$\lambda_{A}^{+}, \lambda_{A}^{-} \quad$ Control incentive/penalty for exceeding/not meeting the nominal energy requirement of adjustable portion of demand. 
$\lambda_{A P}, \lambda_{S I}$ Respective control penalties to ensure smoothest possible scheduling profile is chosen amongst multiple optima for adjustable-pliable and shiftable-interruptible demands

system model that allows it to forecast and, therefore, optimise future system behaviour. In particular, the model should suitably balance the trade-off between accuracy/detail and complexity/tractability. The modelling framework proposed in this paper enables and facilitates the development of advanced control algorithms, such as MPC, to more efficiently manage MES and the flexibility sources they can provide.

It has been proven that flexibility optimisation strategies, which also account for synergies between electricity and heat energy systems, in combination with demand response services and energy storage technologies, have the potential to offer numerous benefits, e.g., providing flexibility to counteract the intermittency of RES. [4]. Currently, to the best of the author's knowledge, no modelling approach that captures all these aspects in a unified framework exists. This need is addressed in this paper which presents a novel, generalised approach to system level modelling of MES for optimisation based energy management problems. The proposed modelling approach is particularly well suited, though not limited, to predictive control applications.

\section{A. Related Literature}

The energy hub has been a prevalent concept for the modelling of MES over the last decade. Energy hubs are described as units with inputs and outputs in which the storage and conversion of different energy carriers take place [5]. In the original formulation, a hub is comprised of converter and storage elements. Converter elements represent how efficiently energy carriers are converted from one form to another, whilst storage elements represent energy storage devices. An excellent resource, containing software tools for energy hub modelling can be found in [6]. The energy hub modelling approach has been applied to a variety operational challenges associated with MES such as expansion planning [7] and [8], optimal design [9] and [10], resource optimisation [11] and [12] and reliability assessment [13]. The energy hub is capable of representing MES flexibility provided by energy storage and the increased degrees of freedom in the energy resource dispatch. However, as highlighted and addressed in [14], existing energy hub approaches do not allow the modelling of arbitrarily complex converter topologies (e.g. including bidirectional power flow and splitting of energy flow at any point in the converter arrangement). Recent contributions based on the energy hub that also aim to address some of these issues are [15] and [16]. However, while both address the issue of arbitrarily complex uni-directional flow paths across an energy hub, neither address the possibility of bi-directional power flow through, or within a hub as is the case with the approach proposed herein.

Alternative approaches to MES modelling are included in [17], [18], [19], [20] and [21]. The models proposed in [17] and [18] are not presented as a generalised approaches for MES, focusing on specific technologies instead, and do not including detailed models of flexible energy demand/production. Generalised MES model is presented in [19] that incorporates spatio-temporal dependencies in a multi-objective optimisation for planning, design and operation of MES. However, the 
emphasis on planning and design as well as the lack of detailed flexible prosumer models and multi-mode devices make it unsuitable for the control-oriented applications considered in this paper. The model presented in [20], while presenting a more general framework based on game theory, is focused on system design with an emphasis on stakeholder benefit over the lifetime of a MES project and is not concerned with day-today or intra-day operation as is typically the case with control focused applications. Also, similarly to [17], [18] and [19], flexible energy demand/production models are not included. A more general approach to MES modelling, considering aspects of control is proposed in [21] where energy systems are described using three fundamental components that exchange both energy and information. However, while the so-called client modules have the potential to model some demand flexibility, they are not able to capture some of the more detailed flexibility characteristics e.g. to shift or interrupt a particular demand profile.

Due to the lack or insufficiency of flexible energy demand/production models, the aforementioned approaches do not capture the full range of flexibility benefits that result from considering MES. In particular, existing methods do not capture the full range of flexibility offered through integrated demand side management (IDSM) which extends the domain of DSM to MES, where in addition to shifting or modulating their energy use, which is realised by DSM, energy users are able to switch the primary source of the consumed energy [22]. Contributions focusing on IDSM include [23], [24] and [25]. However, these are not fully comprehensive approaches because they either focus on demand response (DR) in the cases of [23] and [24], which is a sub-set of DSM/IDSM and relates to the DSM programs implemented to alter energy use in response to particular events such as periods of poor system reliability or high wholesale energy cost, or they focus on specific energy vectors as is the case in [25].

The application of optimisation-based DSM techniques in electrical power systems has been widely researched topic, with models relating to electrically powered resources being recently proposed in [26], [27] and [28]. However, since these approaches focus on electrical energy systems, they do not include multi-energy technologies that unlock additional flexibility and enable IDSM, such as co-generation. It is worth mentioning that the DSM model in [27] covers a range of flexible demand characteristics captured by the model presented in this paper but is only focused on a single primary energy vector.

IDSM and the energy hub modelling approach have been combined in [29], [30] and [31]. These references all include flexible demand models of varying detail, but none offer a fully comprehensive approach to modelling all the possible flexibility characteristics in MES. Therefore, these approaches cannot realise the opportunities from fully exploiting all the possible flexibility characteristics that arise in controllable energy demand/production. In [29], the DR model allows modulation of the demand profile but does not consider the case where the demand profile (or at least a portion of it) is fixed and can only be shifted in time. The DR model in [30] only considers shiftable and curtailable demands while the model proposed in [31] cannot model either fully adjustable or interruptible demands.

Applications of IDSM within MES are considered for the specific case of microgrid operation with DR capabilities in [32], [33] and [34]. However, these contributions only consider curtailable and/or shiftable demands.

Most of the references listed thus far do not address the control problem of automatically operating MES in the presence of uncertain disturbances. The exceptions are [33] and [34], both of which include operational considerations. Also, MPC applications to MES have been reported in [35], [36], [37] and [38]. However, these approaches either do not include IDSM (which is the case in [35]) or the proposed models omit at least some of the flexibility characteristics included in the framework proposed in this paper, i.e. [36] and [38] do not include interruptible demands and [37] only considers adjustable demands based on thermal dynamics associated with buildings.

\section{B. Summary of Contribution}

To the best of the authors' knowledge, no formalised approach to MES modelling for control applications has yet been proposed that incorporates the full range of flexibility sources available in MES. In order to address this, an innovative generalised approach to modelling MES for predictive control design is presented in this paper. The contributions of the paper can be summarised as follows:

- Provision of an innovative energy conversion model that improves on existing approaches (e.g. the energy hub) to facilitate the representation of arbitrarily complex energy converter topologies, i.e. arrangements that can include any number of energy vectors, inputs/outputs, splits/combinations of energy flow, multi-mode devices and/or co-generation devices and bi-directional power flow.

- Proposal of a novel flexible energy demand/production model that, combined with the aforementioned energy conversion model, can capture the full range of flexibility benefits made available through IDSM. While alternative IDSM models have been proposed in the literature, they focus only on a single energy vector and/or none include the full range of flexibility characteristics that can be modelled using the approach proposed herein. Furthermore, the proposed flexible demand/production model can readily incorporate aspects linked to energyuser comfort.

- Unification of MES modelling and IDSM into a new framework that is not based on or restricted to any particular technologies or energy vectors. The modular nature of the framework removes need for spending excessive time developing bespoke mathematical models for particular systems/scenarios as pre-defined components can be connected together to form relatively complex compound models. This aspect makes the approach particularly amenable to software implementation.

- A mechanism for the updating of sequential dispatch schedules and inter-temporal constraints relating to physical dynamic relationships (e.g. storage, thermal loads) 
in order to facilitate on-line application rather than static 'look-ahead' optimisation.

While the proposed modelling approach has been developed primarily for the deployment within predictive control formulations, it can be readily applied to alternative optimisation based energy management applications. For example, developed causal models of MES can be used to evaluate the impact of various 'what-if' scenarios for the purposes of risk analysis, planning, process design (e.g. determining the optimal size of storage or energy conversion device), economic dispatch and unit commitment. Hence, the proposed framework should be of interest to the wider multi-physics and engineering community. Additionally, the proposed modelling approach places no restriction on the particular objective function employed in the optimisation, allowing functions other than that employed for the case study in Section VI-B to be used in other applications (e.g. flexibility optimisation, environmental concerns, ancillary service provision etc.). Alternative functions can also be combined for the purposes of multi-objective optimisation. Finally, the proposed modelling framework can be incorporated into stochastic and/or robust optimisation formulations that rigorously address inevitable uncertainties, such as fixed demand profiles, system model parameters and energy prices.

Further discussion highlighting the superiority of the proposed approach over existing methods is provided in Section VI.

\section{Outline of the Paper}

The remainder of this paper is organised as follows. In Section II an overview of the control oriented modelling tool is provided and the key components of the proposed MES model are introduced. The first of these components, namely the energy conversion model, is described in detail in Section III. In Section IV the storage model component is described. A prosumer model component is described in Section $\mathrm{V}$ along with a detailed description of the flexible demand modelling approach and its extension to energy producers. In Section VI, a particular case-study is introduced that illustrates capabilities of the proposed modelling approach when integrated within MPC control framework. The simulated results relating to the case study are presented in Section VII before the paper is concluded and discussion of future work is provided in Section VIII.

\section{Control Oriented Modelling Framework for Multi-EnERGy Systems}

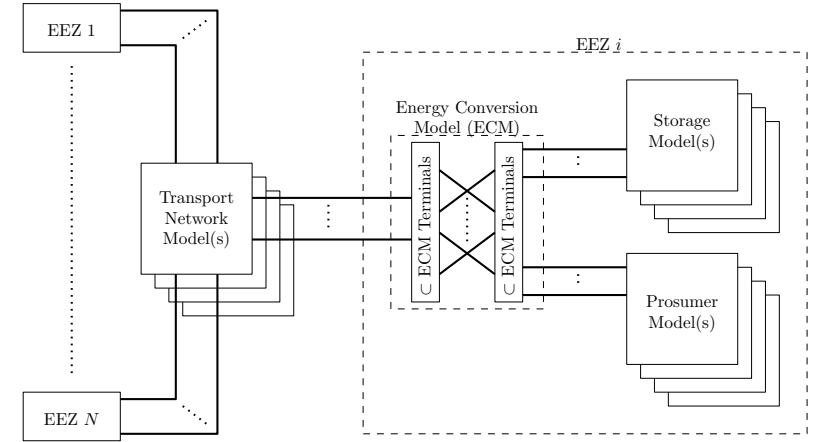

Figure 1: General Model of Multi-EEZ MES

The MES modelling approach proposed in this paper assumes that for certain sections of MES it is reasonable to represent the transportation networks of the various energy carriers within the section as non-dynamic 'lossy' connections. Such sections are termed energy exchange zones (EEZ) and extend the well known energy hub concept [39]. As with the energy hub, the spatial boundaries of a particular EEZ depend upon a given application (e.g. a building, district, city etc.) and are essentially defined by the validity of assuming non-dynamic and linear relationships in the transportation networks. An EEZ contains three types of components, which are the energy conversion model (ECM), the storage model (SM) and prosumer model (PM). The flow, conversion and splitting of the energy carriers are modelled by means of the ECM. Description of various energy storage devices that may exist within an EEZ is provided using the SM. Finally, different types of demand and/or production that may exist within an EEZ are modelled using PM. In a given EEZ, only one ECM component may exist. This is in contrast to SM and PM components, which are not limited by number of occurrences within a single EEZ.

A schematic of a single generic EEZ that exists within a larger multi-zone MES is depicted in Figure 1. The ECM component provides an interface between SM and PM subcomponents in a given EEZ and a wider multi-energy network. Separation of energy conversion, storage dynamics and various prosumer characteristics, as shown in Figure 1, facilitates modular approach to the modelling of MES. Also, the proposed approach for the ECM allows configurable bidirectional converter topologies to be described in a compact manner whilst maintaining a reasonable representation of the underlying physical system. In the following sections, modelling of these three types of EEZ components will be described in greater detail. Whilst the remainder of the paper considers a single EEZ, the proposed modelling framework can be readily applied to general MES composed of multiple EEZs interconnected with transportation networks as indicated in Figure 1.

While EEZ components can be modelled, to some extent, using the existing approaches found in the literature, to the best of the authors' knowledge, detailed models of each have not been combined, formally, into a single general 
modelling framework. Furthermore, the proposed approach includes innovative development of ECM and PM models that provides them with flexibility necessary for the integration within generalised modelling framework. The generality of the propose approach allows it, under certain assumptions, to model any MES that may be comprised by the full set or a subset of the components shown in Figure 1.

\section{ENERGY CONVERSION MODEL}

The ECM, preliminarily introduced in [14], represents a novel extension of the original energy hub concept used as a paradigm for representing energy conversion within MES. In particular, the ability of the ECM modelling approach to represent energy conversion topologies of arbitrary complexity represents one of the key contributions of this paper. No restrictions are imposed on the direction of power flow through a particular arrangement of converters nor the number and/or position of energy carrier splits within. This is achieved whilst still maintaining a reasonable representation of physical conversion devices, with particular reference to multi-output converters such as combined heat and power (CHP) devices. Furthermore, the proposed methodology is well suited to software implementation thus expediting wider model development.

The ECM representation is based on graph theory and is fully characterised by the set of nodes it contains and the associated arcs that provide mutual interconnections between the nodes. Each node of the graph has an energy carrier associated with it. The arcs of the graph represent directional paths through the ECM along which the power can flow and are denoted as $\left(n_{i} \vec{n} n_{j}\right)$ where $n_{i}$ and $n_{j}$ correspond to an arc's source node and sink node, respectively. Each arc has an associated conversion factor, denoted as $\eta_{\left(n_{i} \rightarrow n_{j}\right)}$. The power that flows to $n_{j}$ node is then given by $P_{\left(n_{i} n_{j}\right)}(k) \eta_{\left(n_{i} n_{j}\right)}$, where $P_{\left(n_{i} n_{j}\right)}(k)$ is the power flowing from node $n_{i}$ during sampling period $k$. Also, two index sets are associated with each node. These are $\mathcal{I}_{\rightarrow n_{l}}$, which denotes the index set of source nodes that connect to $n_{l}$, and $\mathcal{I}_{n_{l} \rightarrow \text {, which denotes the }}$ index set of sink nodes that $n_{l}$ connects to.

Each node in the ECM belongs to one of the following four categories: terminal nodes, sum nodes, switch nodes and transmitter nodes. Terminal nodes represent connections of the ECM to externalities that include prosumers, storage elements and the wider energy energy network. Terminal nodes can be further sub-divided into input, output and input/output nodes depending on whether power flows in or out of the ECM. Input nodes have a single outgoing arc and facilitate import of energy to the ECM. Output nodes, conversely, have a single incoming arc and facilitate export of energy out of the ECM. Lastly, input/output nodes have both a single incoming and single outgoing arc connected to the same adjacent node. Input/output nodes allow interface of the ECM with other entities within MES that both import and export energy, e.g. prosumers and storages. The power flowing into or out of the ECM's terminal node $n_{l}$, denoted as $P_{n_{l}}$, is related to the incoming and/or outgoing arc powers according to the following equation:

$$
P_{n_{l}}(k)=\sum_{i \in \mathcal{I} \rightarrow n_{l}} P_{\left(n_{i} \rightarrow n_{l}\right)}(k) \eta_{\left(n_{i} \rightarrow n_{l}\right)}-\sum_{j \in \mathcal{I}_{n_{l}} \rightarrow} P_{\left(n_{l} \rightarrow n_{j}\right)}(k)
$$

Furthermore, non-negativity constraints are imposed on all ECM arc flow variables which ensures that (1) respects a sign convention such that $P_{n_{l}} \geq 0$ for input nodes and $P_{n_{l}} \leq 0$ for output nodes. In addition, upper bounds on arc decision variables can be employed to limit capacity of particular conversion devices.

Sum nodes describe the combining and splitting of a particular energy carrier within the ECM. For each sum node the algebraic sum of incoming and outgoing power flows is equal to zero:

$$
\sum_{i \in \mathcal{I} \rightarrow n_{l}} P_{\left(n_{i} n_{l}\right)}(k) \eta_{\left(n_{i} n_{l}\right)}-\sum_{j \in \mathcal{I}_{n_{l}} \rightarrow} P_{\left(n_{l} n_{j}\right)}(k)=0
$$

Sum nodes represent junctions of energy flow and can be used to describe particular parts of a multi-energy system, such as an electrical busbar or pipework manifold.

Switch nodes, similarly to sum nodes, obey the relationship between their incoming and outgoing arcs given in (2). However, additional binary decision variables, denoted as $\delta$, are associated with each outgoing arc of a switch node to obey the condition (3a) and the constraint (3b) which ensure that only a single outgoing arc is active at any instant in time.

$$
\begin{aligned}
P_{\left(n_{l} n_{j}\right)}(k)>0 \Longleftrightarrow \delta_{\left(n_{l} n_{j}\right)}(k)=1 \quad \forall j \in \mathcal{I}_{n_{l} \rightarrow} & \Longleftrightarrow \sum_{j \in \mathcal{I}_{n_{l}} \rightarrow} \delta_{\left(n_{l} \rightarrow n_{j}\right)}(k) \leq 1
\end{aligned}
$$

Therefore, switch nodes facilitate modelling of multi-mode devices, such as a heat pump that can operate in both heating and cooling modes.

Transmitter nodes are used to model the conversion of energy through a co-generation plant, such as CHP, by requiring that each of their outgoing arc flow values are equal to the sum of the incoming arc flow values, as described in (4). For each transmitter node there are as many equations as there are outgoing arcs.

$$
P_{\left(n_{l} n_{j}\right)}(k)=\sum_{i \in \mathcal{I} \rightarrow n_{l}} P_{\left(n_{i} n_{l}\right)}(k) \eta_{\left(n_{i} n_{l}\right)} \quad \forall j \in \mathcal{I}_{n_{l} \rightarrow}
$$

To prevent the unrealistic case of simultaneous power flow in two parallel but directionally opposite arcs between two nodes, binary decision variables are associated with the particular arcs that obey an equivalent condition to (3a) and the following mutual exclusivity constraint:

$$
\delta_{\left(n_{i} n_{j}\right)}(k)+\delta_{\left(n_{j} n_{i}\right)}(k) \leq 1
$$

\section{Storage Model}

The modular nature of the proposed approach allows for the storage dynamics to be represented by a general $\mathrm{n}$-th order discrete-time state space model, i.e. $\mathbf{x}(k+1)=\mathbf{A} \mathbf{x}(k)+$ 
$\mathbf{B u}(k) ; \mathbf{y}(k)=\mathbf{C x}(k)+\mathbf{D u}(k)$. Note that the generality of state-space models allows multi-input, multi-output SM to be considered. Also, note that the sampling time for a particular application must depend on the dominant time constant of the storage dynamics and be sufficiently small to capture any transient effects. However, this choice must made with respect to the speed of the dynamics that are neglected in the wider framework i.e. if the sampling time is too short, then the steady-state assumptions applied to the rest of the model no longer hold.

Inputs to a SM generally consist of charging/discharging power, denoted as $v(k)$, as well as $N_{w}$ other disturbance inputs, denoted as $\mathbf{w}(k) \in \mathbb{R}^{N_{w}}$, that affect storage, i.e. $\mathbf{u}(k)=\left[v(k) \mathbf{w}(k)^{T}\right]^{T}$. The output of SM, denoted as $y(k)$, represents the stored energy at time $k$. Storage models are interfaced with the rest of the MES via ECM's terminal input/output nodes. Equation (6) indicates how the interface between a SM and ECM is accomplished by setting SM input $v(k)$ equal to the negated node power of the associated terminal node of the ECM, in order to maintain the adopted sign convention. Thus, the power associated with the terminal input/output node of ECM to which a SM is connected is positive during discharging and negative during charging. Also, as SMs are connected to terminal nodes, (5) ensures that simultaneous charging and discharging does not occur.

$$
v(k)=-P_{n_{i}}(k)
$$

Charging/discharging capacities can be limited by implementing upper bounds on the relevant arc decision variables as described in Section III while finite energy capacity is limited by imposing bounds on the stored energy output state of the model, i.e.

$$
\underline{y}(k) \leq y(k) \leq \bar{y}(k)
$$

where $y$ and $\bar{y}$ are the respective lower and upper bounds.

\section{Prosumer Model}

In the wider context of energy systems and the 'smart grid', the prosumer is defined as an energy user that both produces and consumes energy, i.e. producer-consumer. As such, within the context of the proposed modelling framework, the prosumer model (PM) represents a terminus of the MES where energy can be consumed and/or produced. Crucially, the model incorporates additional degrees of freedom allowing it to model dispatchable generation and/or IDSM.

The PM is represented by a set of components. Each component models a particular type of energy demand or production (e.g. local generator or RES). A particular PM is denoted, $p_{j, n_{i}}$, where $j$ is an index corresponding to the PM when more than one is connected to a terminal ECM node $n_{i}$. The net energy demand of a particular prosumer at sampling instant $k$, denoted as $P_{p_{j, n_{i}}}(k)$ is given by (8).

$$
P_{p_{j, n_{i}}}(k)=\sum_{m=1}^{N_{G, p_{j, n_{i}}}} G_{m}(k)-\sum_{n=1}^{N_{L, p_{j, n_{i}}}} L_{n}(k)
$$

Where $G_{m}(k) \geq 0$ and $L_{n}(k) \geq 0$ represent prosumer's $m^{t h}$ generating component and $n^{\text {th }}$ load component, respectively.
Also, $N_{G, p_{j, n_{i}}}$ is the total number of energy generators within the PM and $N_{L, p_{j, n}}$ is the total number of energy consumers. The sign of $P_{p_{j, n_{i}}}(k)$ indicates whether it is producing or consuming, i.e. positive $P_{p_{j, n_{i}}}(k)$ represents production and negative $P_{p_{j, n_{i}}}(k)$ represents consumption. As such, end users that are either sole consumers or producers can be represented by (8) with $G_{m}(k)=0$ or $L_{n}(k)=0$ respectively.

As discussed in the following sub-sections prosumer components can be one of two types: fixed or flexible. In the remainder of this section only consumer type components of the PM will be considered since the potential flexibility of generation type components can be described by using the same approach.

\section{A. Fixed Demand}

Fixed demand components represent energy consumption that cannot be modified either in the rate or time of usage and, therefore, must be met completely and instantaneously. Examples include electrical demand for lighting and television as well as 'on-demand' heating to produce hot water drawn from a tap. Within the proposed framework decision variables are included to represent the consumption with their values fixed at each sampling instant by equality constraints.

\section{B. Flexible Demand}

Each flexible demand component (FDC) is characterised by a particular schedule of its associated decision variables over a future scheduling horizon with length equal to $N_{H} \in \mathbb{I}$ and divided into individual scheduling periods, denoted as $h \in\left\{0,1, \ldots, N_{H}-1\right\}$. Also, it is assumed that the values of the decision variables are constant during each scheduling period. FDC are classified using four different flexibility characteristics:

- Shiftable: Commencement of the demand is not fixed and can be moved within the scheduling horizon but no interruptions are allowed.

- Adjustable: Baseline energy demand can be exceeded or curtailed at particular sampling instants in the scheduling horizon.

- Pliable: The energy demand profile can be manipulated through the scheduling horizon, provided the total energy meets its requirement.

- Interruptible: Energy demand, once commenced at some fixed scheduling period, can be paused between particular sampling instants in the scheduling horizon.

Figure 2 shows how the four flexibility characteristics can be combined to represent different types of demand. In this paper the model representing the combination of shiftable adjustable pliable interruptible (SAPI) demand and shown in the centre of Figure 2 will be described in detail. The other combinations in Figure 2 are special cases of the SAPI demand that result in simplified versions of the model. The model is based on [40], with novel extensions and developments including the adoption of slack decision variables and soft constraints to quantify mismatches to the baseline energy requirements for loads. In particular, the slack variables allow for an explicit 


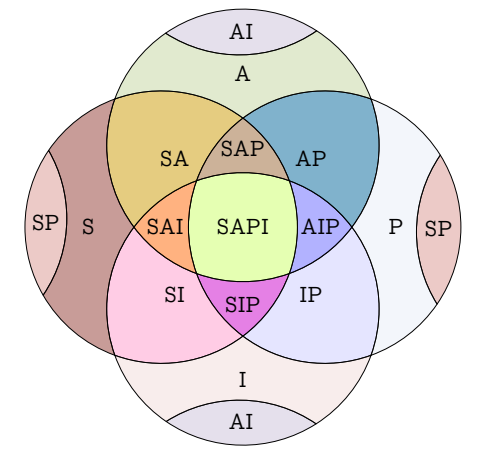

S - Shiftable

A - Adjustable

$\mathrm{P}$ - Pliable

I - Interruptible

Figure 2: Classification of Flexible Demand Types

link to comfort based adjustable loads that require additional dynamic models for their inclusion. Due to space limitations, this feature of the modelling framework is excluded from this paper. However, an example can be found in the associated technical report [41].

In the proposed modelling framework the time-profile of the energy consumption for a particular demand $n$ is split into $N_{n}$ segments that, once started, cannot be interrupted. Energy consumption of the $i^{\text {th }}$ segment of demand $n$ during time period $h$ is denoted as $l_{n, i}(h)$ and is given by:

$$
l_{n, i}(h)=\tilde{l}_{n, i}(h)+\Delta l_{n, i}^{+}(h)-\Delta l_{n, i}^{-}(h) \quad \forall n, i, h
$$

where $\tilde{l}_{n, i}(h)$ is the energy consumption related to the baseline energy requirement for the $i^{\text {th }}$ segment of demand $n$ during time period $h$, and $\Delta l_{n, i}^{+}(h)$ and $\Delta l_{n, i}^{-}(h)$ are adjustable flexibility slack variables that quantify the amount of energy for the demand $n$ that has been curtailed or exceeded, respectively, for each segment $i$ during each time period $h$ effectively relaxing the requirement to meet the entire baseline energy demand at all times as required by the inequalities in (10). The baseline energy requirement represents a preferred amount of energy to be consumed for each segment if adjustable flexibility is not required. The cumulative sum over the scheduling horizon of the baseline energy consumption for the $i^{t h}$ segment of demand $n$ must remain within the baseline lower and upper bounds of the segment's total energy requirement, denoted $\underline{E}_{n, i}$ and $\bar{E}_{n, i}$ respectively:

$$
\underline{E}_{n, i} \leq \sum_{h=0}^{N_{H}-1} \tilde{l}_{n, i}(h) \leq \bar{E}_{n, i} \quad \forall n, i
$$

In the cases of shiftable and/or interruptible and/or adjustable demand type the baseline segment time-profile is fixed and is not a degree of freedom. For these cases $\underline{E}_{n, i}=\bar{E}_{n, i}=E_{n, i}$ and (10) collapses to an equality constraint. Conversely, in the case of any demand that includes pliable type flexibility, the baseline time-profile is not pre-determined and represents an additional degree of freedom that is determined whilst respecting the constraint imposed on the baseline total energy requirement specified in (10). Furthermore, since the total energy requirement is fixed in the cases of shiftable and/or interruptable and/or pliable demand types, its curtailment/increase is not allowed and hence $\Delta l_{n, i}^{+}(h)=0 \forall i, h$ and $\Delta l_{n, i}^{-}(h)=0 \forall i, h$. However, in the case of a flexible demand that combines adjustable flexibility with any of the other three types, $\Delta l_{n, i}^{+}$and $\Delta l_{n, i}^{-}$represent degrees of freedom. Hence, in order to be able to represent any combination of the four demand types, degrees of freedom include the baseline energy consumption time-profile, given by $\tilde{l}_{n, i}(h)$, as well as both curtailment and increase in energy demand, given by $\Delta l_{n, i}^{-}(h)$ and $\Delta l_{n, i}^{+}(h)$ respectively. In the case of adjustable flexibility characteristic both demand curtailment $\left(\Delta l_{n, i}^{-}(h)\right)$ and increase $\left(\Delta l_{n, i}^{+}(h)\right)$ are manipulated through appropriate cost-function selection, i.e. equally large penalties must be imposed on each variable under 'normal' operation, when neither demand curtailment or increase is required, and only one the responses (curtailment or increase) be incentivised at any given $h$.

The resulting energy consumption of a particular demand $n$ at a given time $h$ is represented by $L_{n}(h)$ and is related to the actual energy consumption of its energy segments in the following way:

$$
L_{n}(h)=\sum_{i=1}^{N_{n}} l_{n, i}(h) \quad \forall n
$$

An additional three sets of binary decision variables are utilised to commence or to halt individual demand segments. The first set, $\delta_{\mathbf{n}}^{\mathbf{p}}$, contains segment processing variables which determine whether or not an energy segment $i$ is to be processed during each time step $h$. In particular, $\delta_{n, i}^{p}(h)=1$ if and only if energy segment $i$ is in process at time $h$. The second set, $\delta_{\mathbf{n}}^{\mathrm{c}}$, is comprised of segment complete variables which indicate whether or not a particular energy segment has been completed. More specifically, $\delta_{n, i}^{c}(h)=1$ if and only if segment $i$ has been completed before time step $h$. The third set, $\delta^{\mathbf{w}}$, consists of segment waiting variables which indicate whether or not a demand has been halted between completed and yet-to-be-completed segments i.e. $\delta_{n, i}^{w}(h)=1$ if and only if energy segment $i-1$ has been completed and segment $i$ is waiting to begin.

The seven sets of decision variables that are associated with the general SAPI FDC are listed in (12).

The inequalities relating to the auxiliary variables are provided in (13) where (13a) and (13b) determine the (hard) limits on the size of the energy requirement deviation variables $\Delta l_{n, i}^{+}$and $\Delta l_{n, i}^{-}$, with ${\overline{\Delta l^{+}}}_{n, i}$ and ${\overline{\Delta l^{-}}}_{n, i}$ representing the corresponding upper bounds. Similarly, (13c) determine the minimum $\underline{l}_{n, u}$ and maximum $\bar{l}_{n, u}$ amount of energy that can be scheduled for any particular demand segment at a particular instant. In (13a)-(13c), the multiplication of the segment processing binary decision variables by the lower and/or upper bounds ensures that the corresponding continuous decision variable cannot be non-zero unless the particular segment $i$ is currently under way. The length of a particular demand segment, given in terms of a number of scheduling periods, is constrained from below and above by $\underline{N}_{n, i}^{p}$ and 


$$
\begin{aligned}
\mathbf{l}_{n} & =\left[\left[l_{n, 1}(0), \ldots, l_{n, 1}\left(N_{H}-1\right)\right]^{T}, \ldots,\left[l_{n, N_{n}}(0), \ldots, U_{n, N_{n}}\left(N_{H}-1\right)\right]^{T}\right]^{T} \in \mathbb{R}_{\geq 0}^{N_{n} \cdot N_{H}} \\
\boldsymbol{\delta}_{n}^{\mathbf{p}} & =\left[\left[\delta_{n, 1}^{p}(0), \ldots, \delta_{n, 1}^{p}\left(N_{H}-1\right)\right]^{T}, \ldots,\left[\delta_{n, N_{n}}^{p}(0), \ldots, \delta_{n, N_{n}}^{p}\left(N_{H}-1\right)\right]^{T}\right]^{T} \in \mathbb{I}_{\{0: 1\}}^{N_{n} \cdot N_{H}} \\
\boldsymbol{\delta}_{n}^{\mathbf{c}} & =\left[\left[\delta_{n, 1}^{c}(0), \ldots, \delta_{n, 1}^{c}\left(N_{H}-1\right)\right]^{T}, \ldots,\left[\delta_{n, N_{n}}^{c}(0), \ldots, \delta_{n, N_{n}}^{c}\left(N_{H}-1\right)\right]^{T}\right]^{T} \in \mathbb{I}_{\{0: 1\}}^{N_{n} \cdot N_{H}} \\
\boldsymbol{\delta}_{n}^{\mathbf{w}} & =\left[\left[\delta_{n, 2}^{w}(0), \ldots, \delta_{n, 2}^{w}\left(N_{H}-1\right)\right]^{T}, \ldots,\left[\delta_{n, N_{n}}^{w}(0), \ldots, \delta_{n, N_{n}}^{w}\left(N_{H}-1\right)\right]^{T}\right]^{T} \in \mathbb{I}_{\{0: 1\}}^{\left(N_{n}-1\right) \cdot N_{H}} \\
\tilde{\mathbf{l}}_{n} & =\left[\left[\tilde{l}_{n, 1}(0), \ldots, \tilde{l}_{n, 1}\left(N_{H}-1\right)\right]^{T}, \ldots,\left[\tilde{l}_{n, N_{n}-1}(0), \ldots, \tilde{l}_{n, N_{n}-1}\left(N_{H}-1\right)\right]^{T}\right]^{T} \in \mathbb{R}_{\geq 0}^{N_{n} \cdot N_{H}} \\
\mathbf{l}_{n}^{+} & =\left[\left[\Delta l_{n, 1}^{+}(0), \ldots, \Delta l_{n, 1}^{+}\left(N_{H}-1\right)\right]^{T}, \ldots,\left[\Delta l_{n, N_{n}-1}^{+}(0), \ldots, \Delta l_{n, N_{n}-1}^{+}\left(N_{H}-1\right)\right]^{T}\right]^{T} \in \mathbb{R}_{\geq 0}^{N_{n} \cdot N_{H}} \\
\boldsymbol{\Delta l}_{n}^{-} & =\left[\left[\Delta l_{n, 1}^{-}(0), \ldots, \Delta l_{n, 1}^{-}\left(N_{H}-1\right)\right]^{T}, \ldots,\left[\Delta l_{n, N_{n}-1}^{-}(0), \ldots, \Delta l_{n, N_{n}-1}^{-}\left(N_{H}-1\right)\right]^{T}\right]^{T} \in \mathbb{R}_{\geq 0}^{N_{n} \cdot N_{H}} \\
\mathbf{L}_{n} & =\left[L_{n}(0), \ldots, L_{n}\left(N_{H}-1\right)\right]^{T} \in \mathbb{R}_{\geq 0}^{N_{H}}
\end{aligned}
$$

$\bar{N}_{n, i}^{p}$ respectively.

$$
\begin{aligned}
& 0 \leq \Delta l_{n, i}^{-}(h) \leq \overline{\Delta l^{-}}{ }_{n, i}(h) \delta_{n, i}^{p}(h) \\
& \forall n, i, h \\
& 0 \leq \Delta l_{n, i}^{+}(h) \leq \overline{\Delta l^{+}}{ }_{n, i}(h) \delta_{n, i}^{p}(h) \\
& \quad \forall n, i, h \\
& \underline{l}_{n, i}(h) \delta_{n, i}^{p}(h) \leq l_{n, i}(h) \\
& \quad \leq \bar{l}_{n, i}(h) \delta_{n, i}^{p}(h) \quad \forall n, i, h \\
& \underline{N}_{n, i}^{p} \leq \sum_{h=0}^{N_{H}-1} \delta_{n, i}^{p}(h) \leq \bar{N}_{n, i}^{p} \quad \forall n, i
\end{aligned}
$$

The following inequalities imposed on segment processing and segment complete binary decision variables ensure that once an energy segment has commenced, it is not interrupted and must run to completion:

$$
\begin{aligned}
& \delta_{n, i}^{p}(h)+\delta_{n, i}^{c}(h) \leq 1 \\
& \forall n, i, h \\
& \delta_{n, i}^{p}(h-1)-\delta_{n, i}^{p}(h) \leq \delta_{n, i}^{c}(h) \\
& \forall n, i \quad \forall h \in\left\{1: N_{H}-1\right\} \\
& \delta_{n, i}^{c}(h-1) \leq \delta_{n, i}^{c}(h) \\
& \forall n, i \quad \forall h \in\left\{1: N_{H}-1\right\}
\end{aligned}
$$

The correct sequencing of individual segments is ensured by the following inequality constraint imposed on segment processing and segment complete binary decision variables:

$$
\begin{aligned}
& \delta_{n, i}^{p}(h) \leq \delta_{n, i-1}^{c}(h) \\
& \forall n, h \quad \forall i \in\left\{2: N_{n}\right\}
\end{aligned}
$$

The segment waiting decision variables allow halting of the corresponding energy segment after its predecessor has been completed:

$$
\begin{aligned}
& \delta_{n, i}^{w}(h)=\delta_{n, i-1}^{c}(h)-\left(\delta_{n, i}^{p}(h)+\delta_{n, i}^{c}(h)\right) \\
& \forall n, h \quad \forall i \in\left\{2: N_{n}\right\}
\end{aligned}
$$

The halting period is bounded from below and above by $\underline{N}_{n, i}^{w}$ and $\bar{N}_{n, i}^{w}$ :

$$
\underline{N}_{n, i}^{w} \leq \sum_{h=0}^{N_{H}-1} \delta_{n, i}^{w}(h) \leq \bar{N}_{n, i}^{w} \quad \forall n, h \quad \forall i \in\left\{2: N_{n}\right\}
$$

Finally, (18) specifies the equality constraint which ensures that no segment of demand $n$ can run at particular scheduling instants listed in vector $\mathbf{T}_{n}^{p}$, which correspond to the user time preferences for the demand:

$$
\delta_{n, i}^{p}(h)=0 \quad \forall n, i \quad \forall h \in \mathbf{T}_{n}^{p}
$$

As stated, the other categories of flexible demand shown in Figure 2 are special cases of the SAPI. In particular, the constraints described above take a particular form and not all the decision variable vectors in (12a)-(12h) are required, depending on the classification. For example, S, P and I demands do not require the slack variables $\Delta \mathrm{l}^{+}$and $\Delta \mathrm{l}^{-}$. Due to space limitations, the mathematical description of the fundamental demand types S,A,P and I are not covered in this paper. However, the interested reader is referred to the technical report, which includes more detailed descriptions and illustrations associated with each of the different fundamental demand types [41].

If the above constraints, which model the allowable behaviour of a FDC, are part of an optimisation problem solved repeatedly by a predictive controller (i.e. in an MPC scheme), it is then necessary to account for the effect of the control actions determined by the solution of the previous optimisation (at $k-1)$ on the constraints of the current optimisation $(k)$, a detailed description of which is also provided in [41].

\section{CASe Study}

The MES used to demonstrate the modelling framework presented in Sections II-V is schematically shown in Figure 3. It represents energy infrastructure for three buildings located at the University of Manchester (UoM) North Campus, namely Barnes Wallis Building (BWB), Sackville Street Building (SSB) and Ferranti Building (FB). The transportation networks 
interconnecting the energy infrastructure of the three buildings is assumed to be non-dynamic and, therefore, is considered as a single EEZ introduced in Section II. It can be seen that multiple facets of MES are included within the EEZ: multiple energy vectors (electricity, gas, heat and cooling), multi-generation (CHP), multi-mode operation (heat pump), energy storage (battery and thermal store), bi-directional flow (grid connection and heat network between FB and BWB), prosumer (FB both consumes electricity and produces it from a PV installation) and IDSM with a shiftable-interruptible (SI) electricity demand in SSB, and an adjustable-pliable (AP) heat demand in FB. Not all of the technology shown in Figure 3 currently exists within the actual buildings. In particular, the CHP, heat pump, heat storage, chiller and FDC have all been added to the simulation for demonstration purposes.

The particular MES considered in this case study has been chosen as it demonstrates most features of the modelling framework as well as highlighting the superiority of the proposed approach over existing methods. In particular, it is not possible to represent the energy conversion topology, shown in Figure 3, as a single hub using existing energy hub approaches, even using the recent modifications in [15] and [16], due to the presence of bi-directional power flow within the arrangement. The non-energy hub approach reported in [21] requires the user to distinguish between inputs and outputs referred to sources and clients in [21] when modelling bidirectional power flow in which case, as described in Section III, it is entirely possible for a given interface to act both as an input and an output. The comprehensive MES approach in [19] does facilitate the modelling of bi-directional power flow but, similarly to that reported in [21], it provides no explicit option for multi-mode devices. Furthermore, none of these approaches are capable of modelling the FDCs of the types shown in Figure 3 (or, more generally, fully flexible prosumers). As mentioned in Section I-A, none of the other contributions that include more detailed flexible demand models for MES (e.g. [29] and [31]), are able to capture all the flexibility characteristics modelled in this paper (i.e. shiftable, adjustable, pliable and/or interruptible flexibility), nor can they be mutually combined (e.g. an AP demand can be modelled by the approach in [38] but a SI demand cannot). Another novel aspect of the proposed approach, and one that is not considered already published methods is the procedure of updating receding horizon constraint updates as mentioned in Section V).

The graph diagram that represents the ECM is shown in Figure 4. With reference to Section III, the terminal nodes are represented as squares, sum nodes as circles, transmitter nodes as triangles and switch nodes as irregular pentagons. The alphabetic characters in the node identifiers seen in Figure 4 relate nodes to specific energy carriers (i.e. $e$ is electricity, $g$ is gas, $c$ is cooling and $h$ is heat) which, combined with the numeric characters, generate unique indices for each node. For example, employing the node identifiers in Figure 4, the arc power flow variable from node $g 2$ to $c 1$ is denoted $P_{(g 2 \rightarrow c 1)}$ and the associated arc weighting is denoted $\eta_{(g 2 \rightarrow c 1)}$. In Figure 4, where conversion devices exist in the ECM and consequentially the arc weightings are not equal to 1 , the $\eta$

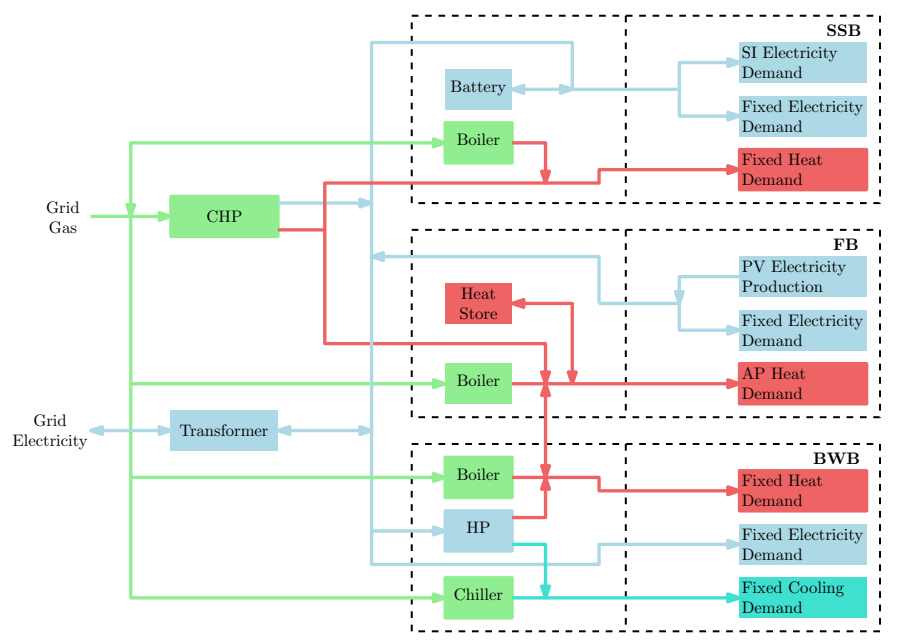

Figure 3: North Campus Buildings Energy System Schematic

symbols associated with particular devices have been labelled. For example, $\eta_{(g 2 \rightarrow c 1)}=\eta_{g c}^{C}$ represents the gas-to-cooling conversion efficiency of the chiller. The values associated with these conversion factors used for the simulations can be seen in Table I. The full ECM requires a single equation for each non-transmitter node and multiple equations for each transmitter node corresponding to the number of its outgoing arcs. Therefore, $21(19+2)$ equations represent the ECM for this case study.

The nodes $h 5$ and $e 4$ in Figure 4 are the dedicated terminal nodes for the battery and thermal storage devices with the weightings of their incoming and outgoing arcs representing the respective charging and discharging efficiencies of the particular devices. For the case study, simple single state models have been adopted to represent the storage devices. Hence, the A,B,C and D state-space matrices introduced in Section IV become scalars and equal to $S_{n_{i}}, 1,1$ and 0 respectively. For each storage $S_{n_{i}}<1$ represents the standby storage efficiency of the device connected to node $n_{i}$. Also, $u(k)=Q_{n_{i}}(k)$ is a scalar quantity representing the charging/discharging power for each storage device and $y(k)=R_{n_{i}}(k)$ is equal to the stored energy in each device. The particular parameter values for the case study are displayed in Table II.

\section{A. Simulation Data}

The UoM Estates department operates a building management system (BMS) that, among other functions, logs energy usage data at half-hourly intervals. Data relating to a particular energy vector for a particular building may be logged using a variety of physical meters (e.g. an electricity meter might be present at each switchboard) but the data logging software includes virtual meters for each building that logs total energy consumption for each separate energy vector. Therefore, data was collected for the total electricity and heat demands of both buildings, in addition to the amount of energy produced by the PV installation on FB. Because the heat pump and chiller were simulated additions to the case study, it was necessary to synthesise a data set for the cooling demand. Also, to match the frequency of the data logging, the duration of each 
Table I: Conversion Efficiencies Related to Physical Devices where Applicable

\begin{tabular}{|c|c|c|c|c|c|}
\hline ECM Arc Factors & Parameter Label & Device & Conversion & & Value \\
\hline$\eta_{(e 1 \rightarrow e 2)}, \eta_{(e 2 \rightarrow e 1)}$ & $\eta_{e e}^{T}$ & Transformer & electricity & & 0.98 \\
\hline$\eta_{(g 2 \rightarrow h 2)}, \eta_{(g 2 \rightarrow h 3)}, \eta_{(g 2 \rightarrow h 4)}$ & $\eta_{g h}^{B}$ & Boiler & gas to heat & & 0.698 \\
\hline$\eta_{(g 3 \rightarrow h 1)}$ & $\eta_{g h}^{C H P}$ & CHP & gas to heat & & 0.485 \\
\hline$\eta_{(g 3 \rightarrow e 2)}$ & $\eta_{g e}^{C H P}$ & $\mathrm{CHP}$ & gas to electricity & & 0.294 \\
\hline$\eta_{(g 2 \rightarrow c 1)}$ & $\eta_{g c}^{C}$ & Chiller & gas to cooling & & 1.1 \\
\hline \multirow[t]{2}{*}{$\eta_{(e 3 \rightarrow h 4)}$} & $\eta_{e h}^{H P}$ & Heat pump & electricity to heat & (summer) & 3.2 \\
\hline & & & & (autumn) & 2.8 \\
\hline \multirow[t]{2}{*}{$\eta_{(e 3 \rightarrow c 1)}$} & $\eta_{e c}^{H P}$ & Heat pump & electricity to cooling & (summer) & 2.5 \\
\hline & & & & (autumn) & 2.1 \\
\hline$\eta_{(h 3 \rightarrow h 5)}$ & $\eta_{h h}^{+}$ & Heat store & charging efficiency & & 0.95 \\
\hline$\eta_{(h 5 \rightarrow h 3)}$ & $\eta_{h h}^{-}$ & & discharging efficiency & & $1 / 0.95$ \\
\hline$\eta_{(e 2 \rightarrow e 4)}$ & $\eta_{e e}^{+}$ & Battery & charging efficiency & & 0.9 \\
\hline$\eta_{(e 4 \rightarrow e 2)}$ & $\eta_{e e}^{-}$ & & discharging efficiency & & $1 / 0.9$ \\
\hline
\end{tabular}

Table II: Additional Simulation Parameters

\begin{tabular}{ccc|ccc}
\hline Parameter & Description & Value & Parameter & Description & Value \\
$P_{(g 2 \rightarrow g 3)}$ & CHP input capacity & $340 \mathrm{~kW}$ & $P_{(e 2 \rightarrow e 3)}$ & HP input capacity \\
$\underline{R}_{h 5} / \bar{R}_{h 5}$ & Min./max. heat energy storage & $30 / 150 \mathrm{kWh}$ & $\bar{Q}_{h 5} / Q$ & Max. heat store charge/discharge & $100 \mathrm{~kW}$ \\
$\underline{R}_{e 4}, \bar{R}_{e 4}$ & Min./max. battery storage & $27 / 180 \mathrm{kWh}$ & $\bar{Q}_{e 4}, \underline{Q} e 4$ & Max. battery charge/discharge & $225 \mathrm{~kW}$ \\
$S_{h 5}$ & Heat storage standby loss factor & 0.95 & $S_{e 4}$ & Battery standby loss factor & 0.96 \\
\hline
\end{tabular}

scheduling period $h$ is set equal to each sampling period $k$ to result in a half-hourly sampling frequency.

For the purposes of the case study, it was assumed that $60 \%$ of heat energy consumed in the FB was fixed with the remaining $40 \%$ assumed to be adjustable and pliable (AP). A set of energy demand segments was then computed using the $40 \%$ of AP demand by summing the energy consumption over every $2 \mathrm{hr}$ period. Hence the total baseline amount of heat energy delivered to the building remains equal to the original demand profile but the controller has freedom to manipulate that portion of the energy consumption profile according to its objective. This configuration could represent a situation where thermal modelling of a building has previously been conducted and, using historic data, it produced a predicted baseline energy consumption profile over bi-hourly periods. It is also assumed that the AP heat demand, denoted $L_{A P}$, is a continuously running demand. Therefore, the minimum and maximum number of scheduling periods during which each energy segments must be processed are both equal to 4 ( $2 \mathrm{hr}$ time interval) i.e. $\underline{N}_{A P, i}^{p}=\bar{N}_{A P, i}^{p}=4 \forall i$. The maximum amount of energy the AP demand can consume over one sampling period is set to $300 \mathrm{kWh}$ (i.e. $\bar{U}_{A P, i}(k+h)=300 \forall i, k, h$ ). Also, the maximum deviation from the baseline requirement during a sampling period is $\pm 20 \mathrm{kWh}$.

The shiftable and interruptible (SI) electricity demand, denoted $L_{S I}$, represents the energy consumption of a machine located within SSB that has a fixed demand profile each time it runs. The example profile used for the case study is equal to $\mathbf{D}_{S I}=\left[E_{S I, 1}, E_{S I, 2}, E_{S I, 3}, E_{S I, 4}, E_{S I, 5}, E_{S I, 6}, E_{S I, 7}\right]^{T}=$ $[10,10,15,10,15,15,10]^{T}$. Each element of $\mathbf{D}_{S I}$ has $\mathrm{kWh}$ units. Also, $\underline{N}_{S I, i}^{p}=\bar{N}_{S I, i}^{p}=1 \forall i$. Since the demand is interruptible, between any energy segment in the profile the controller is able to delay the commencement of the next segment by up to maximum of 3 scheduling periods (i.e.
$\left.\bar{N}_{S I, i}^{w}=3 \forall i\right)$. Further, as the demand is shiftable, the particular period during which the machine commences its operation can be determined by the controller.

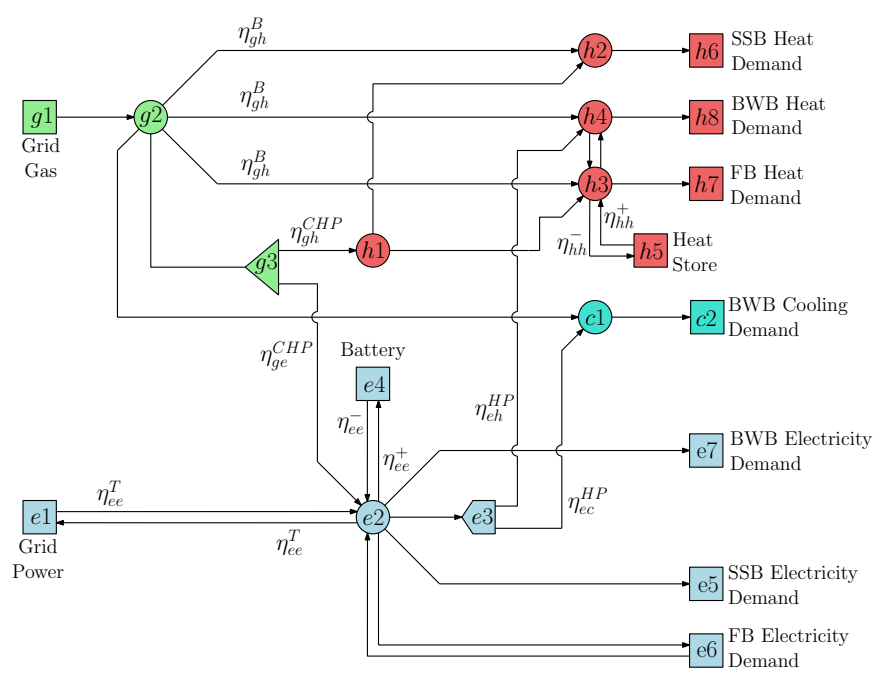

Figure 4: North Campus Buildings Energy Conversion Model

Other parameter values related to the case study are presented in Table II along with their units where applicable.

\section{B. Control Scheme}

In order to exemplify the control-oriented modelling framework presented in this paper, a certainty equivalent model predictive controller (CEMPC), where future uncertainties in the optimisation are assumed deterministic based on predicted values [42], is applied to the case study introduced earlier in this section. The CEMPC is introduced in this section whilst the results of its application are provided in Section VII. 
The CEMPC solves the deterministic multi-period optimisation problem given in (19) during each sampling period where:

$$
\begin{aligned}
V_{\text {cost }}(k+h)= & {\left[P_{(e 1 \rightarrow e 2)}(k+h) \pi_{e}^{\text {buy }}(k+h)\right.} \\
& -P_{(e 2 \rightarrow e 1)}(k+h) \eta_{(e 2 \rightarrow e 1)} \pi_{e}^{\text {sell }}(k+h) \\
& \left.+P_{g 1}(k+h) \pi_{g}^{\text {buy }}(k+h)\right]
\end{aligned}
$$

$$
\begin{aligned}
& V_{A}(k+h)= \\
& \sum_{i=1}^{N_{n}}\left[\Delta l_{A P, i}^{+}(k+h) \lambda_{A}^{+}(k+h)+\Delta l_{A P, i}^{-}(k+h) \lambda_{A}^{-}(k+h)\right]
\end{aligned}
$$

$$
\begin{aligned}
V_{\text {smooth }}(k+h)= \\
\quad\left[L_{S I}(k+h+1)-L_{S I}(k+h)\right]^{2} \lambda_{S I}^{\Delta}(k+h) \\
+\left[L_{A P}(k+h+1)-L_{A P}(k+h)\right]^{2} \lambda_{A P}^{\Delta}(k+h)
\end{aligned}
$$

In (19), $V_{\text {cost }}$ is the economic term of the objective function with $\pi_{e}^{\text {buy }}$ and $\pi_{e}^{\text {sell }}$ representing the purchase price and received revenue for selling electrical energy, respectively. Similarly, $\pi_{g}^{b u y}$ represents the cost of purchasing gas.

The term $V_{A}$ relates to the adjustable portion of demand in the system. Variables $\Delta l_{A P, i}^{+}$and $\Delta l_{A P, i}^{-}$are the slack variables (introduced in Section V-B) for the AP FDC. Also, $\lambda_{A}^{+}$and $\lambda_{A}^{-}$represent a penalty or incentive for respectively exceeding $\left(\Delta l_{A P, i}^{+}>0\right)$ or not meeting $\left(\Delta l_{A P, i}^{-}>0\right)$ the apparent energy requirement of the AP energy segment profile. These parameters can be used to implement the provision of services to system operators e.g. frequency response, demand response etc. During 'normal' operation, where no additional service is being provided, $\lambda_{A}^{+}$and $\lambda_{A}^{-}$take positive values, greater than the maximum $\pi_{e}^{b u y}$ or $\pi_{g}^{b u y}$. Hence, unless it is necessary to meet an energy balancing constraint imposed on the entire EEZ, the deviations will remain equal to zero. If, however, it becomes desirable to deviate from the baseline energy requirement during some period(s) in the control horizon then the corresponding $\lambda_{A}^{+}(k+h)$ or $\lambda_{A}^{-}(k+h)$ will become negative, thus influencing the optimisation to maximise the corresponding deviation variable during the period i.e. if curtailment is required then the corresponding $\lambda_{A}^{-}$will be negative and if an increase in demand is required the corresponding $\lambda_{A}^{+}$will be negative. Also, it is possible to incentivise the operation of a particular energy converter (e.g. the heat pump) by changing the cost coefficient, related to its corresponding variable, into a negative value when, at the same time, the demand response is required.

Finally, the $V_{\text {smooth }}$ term imposes a control performance penalty on the difference in energy consumption of the FDC between consecutive periods in the control horizon. This ensures that in the presence of multiple optimal scheduling solutions of the FDC, a unique solution that produces the smoothest possible energy consumption profile is selected. The control performance penalty is included based on the assumption that it is typically desirable to avoid large ramp rates applied to manipulating variables. To ensure that the control performance penalty does not significantly influence the major economic objective of the controller the corre- 
sponding objective function coefficients are set such that $\lambda_{S I}^{\Delta}, \lambda_{A P}^{\Delta}<<\pi_{e}^{b u y}, \pi_{e}^{b u y}$.

The certainty equivalence of the CEMPC means that future values of uncertain variables, namely fixed energy demand and PV production, are treated as deterministic values in the corresponding MPC optimisation problem. These future values are obtained by utilising an adaptation of a Self Organising Map (SOM) neural network architecture [43]. Note that exploring forecasting techniques is beyond the scope of this paper and the SOM was chosen due to its simplicity in implementation. The SOMs are trained offline using historical data and then, during online implementation, they utilise at each time $k$ the previous $N_{H}-1$ observations, along with the observation at $k$, to predict the future $N_{H}-1$ values of each uncertain variable. These future values of the uncertain variables are then used to solve the deterministic MPC optimisation problem in order to obtain the optimal schedule of control inputs. For the particular case study introduced in Section VI, collected data spanning the period March 2016 - April 2017 was used to train the SOMs to predict future values of energy demand and PV production.

Due to the presence of the binary decision variables in the EEZ model as well as the quadratic term (20c), the corresponding MPC optimisation problem is a mixed integer quadratic program (MIQP) which is solved using the commercial solver CPLEX at each sampling period. For the case study in this paper the resulting optimisation problem is defined with 9790 decision variables of which 3312 are binaries. The average computation time to solve the optimisation problem during each control period is 32 seconds using a general purpose laptop computer.

\section{RESULTS}

Simulations were run, using data from a random week in the autumn of 2017. In order to highlight particular controller responses, an artificial time of use tariff was used for the cost of purchasing electricity from the grid. Fixed prices were used for the purchasing of gas and the export of electricity. The daily price profiles are shown in Figure 5a. The SI demand is

a) Energy Price

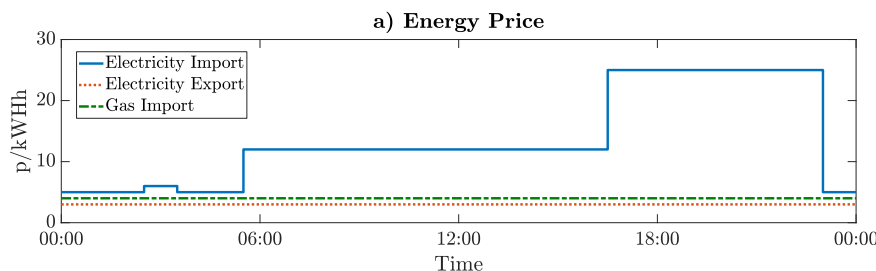

b) SSB Shiftable Interruptible Electricity Demand

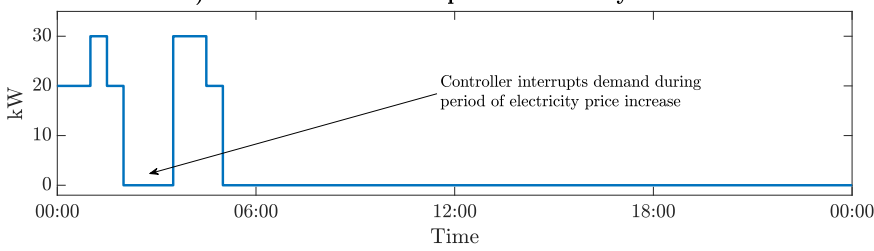

Figure 5: Energy Price Profile for One Day

shown in Figure 5b. It can be seen that the controller schedules the SI demand to run during the period when electricity is least expensive (between 23:00 and 05:30). Also, as there is a slight price increase between 02:30 and 03:30, the controller interrupts the demand for $1.5 \mathrm{hrs}$ to avoid buying electricity at the slightly elevated price. Figures $6 a$ and $6 \mathrm{~b}$ show the battery charging/discharging profile and the stored energy in the battery respectively. The battery is charged through the periods of relatively inexpensive electricity before being discharged once the price is increased at 03:00, 05:30 and 16:30. Figure 7 shows the heat demand of each building
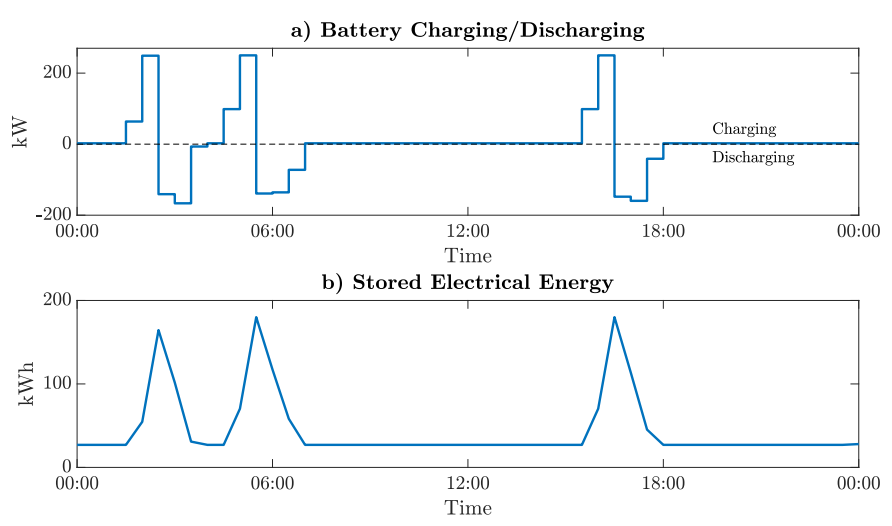

Figure 6: SSB Controllable Demand and Battery Operation

(7a) along with the CHP operation (7b) during an Autumn weekday. As the CHP is sized for base load it runs at capacity throughout the day. For most of the day, the heat produced by the CHP is diverted to SSB as does it not benefit from a heat interconnection with either the heat pump or energy storage. Figure 8 shows the heat dispatch for the BWB (8a) along with the heat energy storage profile ( $8 b)$. The BWB heat dispatch

a) Heat Demand

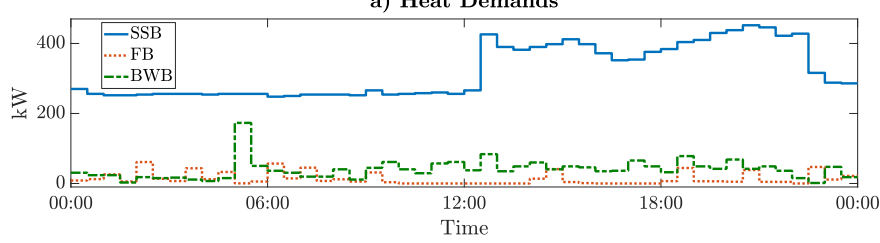

b) CHP Heat Dispatch

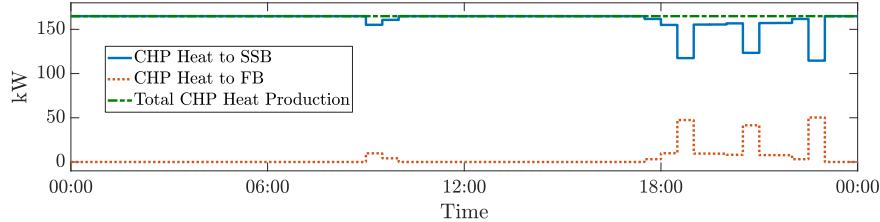

Figure 7: Building Heat Demands and CHP Dispatch

plot shows the HP being operated at maximum capacity until 07:30. It also shows that an excess in heat production is sent to the FB building where the heat store is situated. This is the case except between 05:00 - 05:30 when, coinciding with the peak in heat demand in BWB, heat flow between BWB and FB is reversed and heat storage discharge is sent from FB to BWB which is combined with the HP heat production to meet the increase in heat demand. Between 07:30 and 12:00 the HP operation is reduced in accordance with the reduction in FB heat demand as, combined with the heat store discharge, it is 
sufficient to meet the demands of both FB and BWB. The HP is again operated at maximum capacity from 12:00 to 16:30 and heat is largely exported to FB charge the store, except at 12:30 when another relative peak in BWB heat demand causes heat storage discharge and heat to flow from FB to BWB. At 16:30 the electricity price reaches its premium and, after discharging the heat store, it is more economic to run the boiler in BWB. This also coincides, as shown in Figure 7b, with some of the CHP heat output being diverted from SSB to FB, which avoids the use of FB boiler.
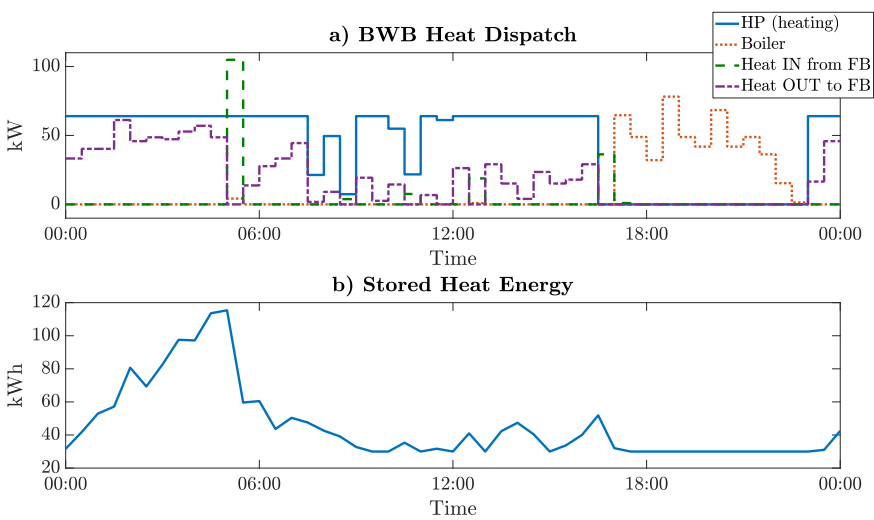

Figure 8: BWB Heat Dispatch and Ferranti Heat Store Operation

In order to demonstrate the features of the AP heat demand in the FB two separate simulations were run. In the first, no incentive was provided at any point for violating the baseline energy requirement. In the second, an incentive was provided to increase the electricity demand of the heat pump between 17:30 and 19:30. Figure 9a shows the fixed heat demand for the FB along with the AP demand profile for both cases (with and without the incentive). Figure 9b shows the BWB heat dispatch for the case when the incentive is applied (the equivalent plot for the case without the incentive is shown in the upper plot of Figure 8a). Also, Figure 9a shows the value of the exceeded energy requirement deviation variable $\Delta \mathrm{l}_{A P, 1}^{+}$. It can be seen that, due to the incentive, additional energy consumption is scheduled during the incentivised period. In particular, as the maxiumum allowable deviation from the baseline energy requirement over any sampling period is \pm 20 $\mathrm{kWh}$, the controller schedules a maximum increase of $80 \mathrm{kWh}$ over the corresponding period. This additional consumption can also be seen in Figure 9a and 9b where the HP is operated during the incentivised period and heat is exported to FB. This is in contrast to the ex-incentive case where only the boiler is operated during this period (see Figure 8a). Simulations were also run for the scenario where the CHP plan was not present in the system for both Summer and Autumn days. The average cost savings for the two days were approximately $50 \%$.

\section{CONCLUSION}

This paper has presented a new general, comprehensive and formalised modelling framework for the representation of multi-energy systems that facilitates the optimisation of a

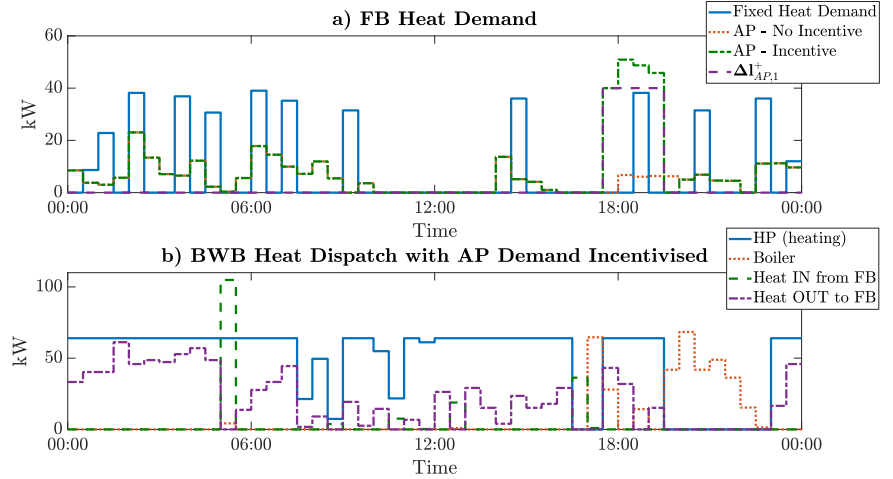

Figure 9: Ferranti AP Heat Demand with Barnes Wallis Heat Dispatch

broad range of flexibility benefits. The framework is particularly suited for, but not restricted to, the design of predictive control strategies. The approach is capable of representing converter topologies of arbitrary complexity that can include any number of energy vectors, inputs/outputs, splits/combinations of energy flow, multi-mode devices and/or co-generation devices and bi-directional power flow.

The modelling tool was used to develop the optimal online energy management for a group of university buildings within a simulation environment. Where possible, actual energy consumption data was used to ensure a representative case-study. The simulation results demonstrated benefits of utilising predictive control to economically optimise operation of a relatively complex multi-energy system.

Future work will focus on utilising the proposed modelling framework to formulate a control scheme with incorporated robustness against various uncertainties, such as fixed demand profiles, system model parameters and energy prices.

\section{REFERENCES}

[1] C. Gellings, "The concept of demand-side management for electric utilities," Proceedings of the IEEE, vol. 73, no. 10, pp. 1468-1470, 1985.

[2] P. Mancarella and G. Chicco, "Integrated energy and ancillary services provision in multi-energy systems," Proceedings of IREP Symposium: Bulk Power System Dynamics and Control - IX Optimization, Security and Control of the Emerging Power Grid, IREP 2013, no. ii, 2013.

[3] J. M. J. M. Maciejowski, Predictive Control: With Constraints. Harlow: Prentice Hall, 2002.

[4] D. Neves, A. Pina, and C. A. Silva, "Demand response modeling: A comparison between tools," Applied Energy, vol. 146, pp. 288-297, 2015.

[5] M. Geidl and G. Andersson, "Optimal power flow of multiple energy carriers," IEEE Transactions on Power Systems, vol. 22, no. 1, pp. 145-155, 2007.

[6] EMPA;University of Victoria, "Holistic urban energy simulation," accessed 2018-02-06. [Online]. Available: https://hues-platform.github.io/

[7] X. Zhang, M. Shahidehpour, A. Alabdulwahab, and A. Abusorrah, "Optimal expansion planning of energy hub with multiple energy infrastructures," IEEE Transactions on Smart Grid, vol. 6, no. 5, pp. 2302-2311, Sep. 2015.

[8] X. Zheng, G. Wu, Y. Qiu, X. Zhan, N. Shah, N. Li, and Y. Zhao, "A minlp multiobjective optimization model for operational planning of a case study cchp system in urban china," Applied Energy, vol. 210, pp. 1126-1140, 2018

[9] P. Gabrielli, M. Gazzani, E. Martelli, and M. Mazzotti, "Optimal design of multienergy systems with seasonal storage," Applied Energy, vol. 212, no. June, p. 720, 2018.

[10] A. Dolatabadi, B. Mohammadi-Ivatloo, M. Abapour, and S. Tohidi, "Optimal stochastic design of wind integrated energy hub," IEEE Transactions on Industrial Informatics, vol. 13, no. 5, pp. 2379-2388, 2017.

[11] J. F. Marquant, R. Evins, L. A. Bollinger, and J. Carmeliet, "A holarchic approach for multi-scale distributed energy system optimisation," Applied Energy, vol. 208, no. May, pp. 935-953, 2017.

[12] W. Lin, X. Jin, Y. Mu, H. Jia, X. Xu, X. Yu, and B. Zhao, "A two-stage multiobjective scheduling method for integrated community energy system," Applied Energy, vol. 216, no. February, pp. 428-441, 2018. 
[13] M. Moeini-Aghtaie, H. Farzin, M. Fotuhi-Firuzabad, and R. Amrollahi, "Generalized analytical approach to assess reliability of renewable-based energy hubs," IEEE Transactions on Power Systems, vol. 32, no. 1, pp. 368-377, Jan. 2017.

14] S. Long, A. Parisio, and O. Marjanovic, "A conversion model for nodes in multienergy systems," 2017 IEEE Manchester PowerTech, pp. 1-6, 2017.

[15] Y. Wang, J. Cheng, N. Zhang, and C. Kang, "Automatic and linearized modeling of energy hub and its flexibility analysis," Applied Energy, vol. 211, no. November 2017, pp. 705-714, 2018.

[16] J. Ramos-Teodoro, F. Rodríguez, M. Berenguel, and J. L. Torres, "Heterogeneous resource management in energy hubs with self-consumption: Contributions an application example," Applied Energy, vol. 229, no. August, pp. 537-550, 2018.

[17] M. Di Somma, B. Yan, N. Bianco, G. Graditi, P. B. Luh, L. Mongibello, and V. Naso, "Multi-objective design optimization of distributed energy systems through cost and exergy assessments," Applied Energy, vol. 204, pp. 1299-1316, 2017.

[18] Y. Jiang, J. Xu, Y. Sun, C. Wei, J. Wang, S. Liao, D. Ke, X. Li, J. Yang, and $\mathrm{X}$. Peng, "Coordinated operation of gas-electricity integrated distribution system with multi-cchp and distributed renewable energy sources," Applied Energy, vol. 211, no. May 2017, pp. 237-248, 2018

[19] S. Samsatli and N. J. Samsatli, "A multi-objective milp model for the design and operation of future integrated multi-vector energy networks capturing detailed spatio-temporal dependencies," Applied Energy, no. July, pp. 1-28, 2017.

[20] Z. Huang, H. Yu, X. Chu, and Z. Peng, "A novel optimization model based on game tree for multi-energy conversion systems," Energy, vol. 150, pp. 109-121, 2018.

[21] C. Fauvel, F. Claveau, P. Chevrel, and P. Fiani, "A flexible design methodology to solve energy management problems," International Journal of Electrical Power and Energy Systems, vol. 97, no. November 2017, pp. 220-232, 2018.

[22] A. Sheikhi, M. Rayati, S. Bahrami, and A. M. Ranjbar, "Integrated demand side management game in smart energy hubs," IEEE Transactions on Smart Grid, vol. 6 , no. 2 , pp. $675-683,2015$.

[23] J. Wang, H. Zhong, Z. Ma, Q. Xia, and C. Kang, "Review and prospect of integrated demand response in the multi-energy system," Applied Energy, vol. 202, no. 51537005 , pp. 772-782, 2017.

[24] S. Bahrami, S. Member, A. Sheikhi, and S. Member, "From demand response in smart grid toward integrated demand response in smart energy hub," IEEE Transactions on Smart Grid, vol. 7, no. 2, pp. 650-658, 2016.

[25] C. Shao, Y. Ding, J. Wang, and Y. Song, "Modeling and integration of flexible demand in heat and electricity integrated energy system," IEEE Transactions on Sustainable Energy, vol. 9, no. 1, pp. 361-370, 2017.

[26] G. R. Aghajani, H. A. Shayanfar, and H. Shayeghi, "Demand side management in a smart micro-grid in the presence of renewable generation and demand response," Energy, vol. 126, pp. 622-637, 2017

[27] S. Nan, M. Zhou, and G. Li, "Optimal residential community demand response scheduling in smart grid," Applied Energy, vol. 210, pp. 1280-1289, 2018.

[28] M. Shafie-khah, N. Mahmoudi, P. Siano, T. K. Saha, and J. P. S. Catalao, "A comprehensive model to integrate emerging resources from supply and demand sides," IEEE Transactions on Smart Grid, vol. 3053, no. c, pp. 1-1, 2017.

[29] S. Pazouki and M. R. Haghifam, "Optimal planning and scheduling of energy hub in presence of wind, storage and demand response under uncertainty," International Journal of Electrical Power and Energy Systems, vol. 80, pp. 219-239, 2016.

[30] F. Brahman, M. Honarmand, and S. Jadid, "Optimal electrical and thermal energy management of a residential energy hub, integrating demand response and energy storage system," Energy and Buildings, vol. 90, pp. 65-75, 2015.

[31] D. Setlhaolo, S. Sichilalu, and J. Zhang, "Residential load management in an energy hub with heat pump water heater," Applied Energy, no. July 2012, pp. 0-1, 2017

[32] M. Jin, W. Feng, P. Liu, C. Marnay, and C. Spanos, "Mod-dr: Microgrid optima dispatch with demand response," Applied Energy, vol. 187, pp. 758-776, 2017.

[33] T. Ma, J. Wu, and L. Hao, "Energy flow modeling and optimal operation analysis of the micro energy grid based on energy hub," Energy Conversion and Management vol. 133, pp. 292-306, 2017

[34] A. Parisio, E. Rikos, G. Tzamalis, and L. Glielmo, "Use of model predictive control for experimental microgrid optimization," Applied Energy, vol. 115, pp. 37-46, Feb. 2014.

[35] N. Holjevac, T. Capuder, N. Zhang, I. Kuzle, and C. Kang, "Corrective receding horizon scheduling of flexible distributed multi-energy microgrids," Applied Energy, 2017.

[36] X. Yang, Y. Zhang, B. Zhao, F. Huang, Y. Chen, and S. Ren, "Optimal energy flow control strategy for a residential energy local network combined with demand-side management and real-time pricing," Energy and Buildings, vol. 150, pp. 177-188, 2017

[37] G. Darivianakis, A. Georghiou, R. S. Smith, and J. Lygeros, "The power of diversity: Data-driven robust predictive control for energy efficient buildings and districts," IEEE Transactions on Control Systems Technology, 2016.

[38] A. Parisio, C. Wiezorek, T. Kyntäjä, J. Elo, K. Strunz, and K. H. Johansson, "Cooperative mpc-based energy management for networked microgrids," IEEE Transactions on Smart Grid, vol. 8, no. 6, pp. 3066-3074, 2017.

[39] M. Geidl and G. Andersson, "A modeling and optimization approach for multiple energy carrier power flow," Power Tech, 2005 IEEE Russia, pp. 1-7, 2005.

[40] K. C. Sou, J. Weimer, H. Sandberg, and K. H. Johansson, "Scheduling smart home appliances using mixed integer linear programming," in IEEE Conference on Decision and Control and European Control Conference. IEEE, Dec. 2011, pp. 5144-5149.

[41] S. Long, O. Marjanovic, and A. Parisio, "A control-oriented modelling tool for multi-energy systems - technical report," Manchester, 2018, accessed: 04-06-2018. [Online]. Available: http://personalpages.manchester.ac.uk/postgrad/sebastian.long/ files/GCOMFMES.pdf
[42] J. Skaf and S. P. Boyd, "Design of affine controllers via convex optimization," IEEE Transactions on Automatic Control, vol. 55, no. 11, pp. 2476-2487, 2010.

[43] G. A. Barreto, "Time series prediction with the self-organizing map: A review," in Perspectives of Neural-Symbolic Integration, ser. Studies in Computational Intelligence, B. Hammer and P. Hitzler, Eds. Berlin, Heidelberg: Springer Berlin Heidelberg, 2007, vol. 77, no. August 2007, pp. 135-158. 